\title{
Value of color doppler ultrasound, kub and urinalysis in diagnosis of renal colic due to ureteral stones
}

\author{
Mahmoud Abdel-Gawad ${ }^{1}$, Ravi Kadasne ${ }^{1}$, Chandrashekar Anjikar ${ }^{1}$, Emad Elsobky² $^{2}$ \\ ${ }^{1}$ Department of Urology, Emirates International Hospital, Al Ainvand ${ }^{2}$ Department of Urology, Al-Noor \\ Hospital, Abu Dhabi, United Arab Emirates
}

\section{ABSTRACT}

Purpose: Despite the routine use of helical CT in diagnosis of renal colic, there are recent concerns regarding the radiation exposure, overuse and costs. We attempted in this retrospective study to evaluate the accuracy of ultrasound (gray-scale and color Doppler with twinkling), KUB and urinalysis in diagnosis of renal colic due to ureteral calculi presented in Emergency Room.

Materials and Methods: A total of 939 consecutive cases of renal colic presented to ER have been managed and evaluated by ureteral ultrasound, KUB and urinalysis for the presence of ureteral stones. Non-confirmatory cases were subjected to Helical CT examination.

Results: Renal and ureteral ultrasound (gray-scale) alone detected ureteral calculi in 615 cases (65.4\%) and after utilizing Color Doppler Ultrasound with twinkling the diagnosis was made with confidence in 935 cases (99.6\%) but 4 (0.4\%). KUB showed radiopaque stones in 503 (53.6\%) patients and no stones were detected in 436 (46.4\%). Microhematuria presented in $835(88.9 \%)$ cases while absent in 102 (10.9\%). There were 190 (20.3\%), 77 (8.2\%) and 671 (71.5\%) patients with upper, middle and lower ureteral stones respectively. The simultaneous positive findings in US and KUB with microhematuria were found only in 453 (48.2\%) cases.

Conclusions: The use of Color Doppler ultrasound with twinkling increased the detection rate of ureteral stones in acute renal colic patients presented to ER with less radiation exposure. Ultrasound examination as a single modality is superior to KUB and urinalysis in initial diagnosis of renal colic.

\section{ARTICLE INFO}

\section{Key words:}

Renal Colic; Ultrasonography;

Calculi; Ureter; Hematuria

Int Braz J Urol. 2014; 40: 513-9

Submitted for publication:

July 26, 2013

Accepted after revision:

December 10, 2013

\section{INTRODUCTION}

Acute flank pain is one of the most common presentations in ER. CT is generally accepted as the imaging study of choice to evaluate patients with flank pain in emergency department (1). In 1995, Smith et al. described the use of helical CT in patients suspected of having acute renal colic (2). Recently, there is growing awareness and concerns about the overuse of CT in evalua- tion of patient with acute flank pain particularly the radiation effects and potential health hazards during follow-up of stones (3). Instead of the recent advancement in US technology, most of the radiologists have difficulty in localization of the ureteric stones especially if the gray-scale ultrasound is not conclusive. The twinkling in color Doppler images is an artifact created by a rapidly changing series of colored horizontal bars that appear beyond the reflex surfaces assuming 
a triangular shape when highly evident (4). Many recent studies have reported and encouraged the use of twinkling sign to improve the accuracy of ultrasound to detect renal and ureteral stones (5). In this retrospective study, we evaluated the use of US (gray-scale and Color Doppler), KUB and urine analysis in diagnosis of renal colic due to ureteral calculi in patients presented to ER.

\section{MATERIALS AND METHODS}

\section{Patients' data}

After approval of Institutional Review Board, we conducted this retrospective study by reviewing the medical records of patients diagnosed with acute renal colic in the emergency room from January 2003 to December 2010. The patients were identified by using ICD-9 codes and were confirmed by charts review. The patients were evaluated for flank pain in the form of general and local examinations. After control of pain, the patient underwent immediate radiological examinations in the form of plain x-ray of the abdomen (KUB) and ultrasound scanning (gray-scale and color Doppler) of both flanks. Microscopic urine examination was performed and more than 3 RBCs/ HPF was considered significant and counted. The findings of KUB were interpreted by two separate independent conventional radiologists who were blinded to the results of the ultrasound findings. US examinations (gray-scale and color Doppler with twinkling) were performed by another two senior ultrasonographists.

\section{Ultrasound Technique}

No bowel preparation or full bladder was mandatory as most of the patients arrived with acute renal colic. All US examinations were performed by using three models of Ultrasound machines that were available during the study period (Toshiba Power Vision 6000, Model: 370A-SSA, Japan, Toshiba Aplio, Model USEL-790A, Japan and Philips iU22 - Bothell WA, USA). A multi-frequency convex abdominal transducer with frequency of 1 to $6 \mathrm{MHz}$ was used. A diagnostic criterion was direct visualization of the calculus in the ureteric lumen with or without associated hydronephrosis. Symptomatic side was screened to assess the degree of hydronephrosis and for possible stones in the kidney, pelvis or upper ureter. If no definite stone was visualized, the bladder and the vesicoureteral junction were screened for possible calculi. If no stone was identified at these sites, the effort was made to trace the ureter from the renal pelvis till the iliac crossing. Graded compression with insinuation helped in localization of the stone at this site by decreasing the distance between the transducer and ureter. Ultrasound scanning of full bladder was not necessary in every case as filling of the bladder was not possible in all patients due to severe pain and possible lower ureteric calculus causing frequency of urination; in addition, an over distended bladder hindered detection and limited the amount of pressure that can be applied to the bladder. Once the stone was identified, the gray-scale ultrasound was used to measure the long axis of the stone and its length was recorded. The location of the stone was recorded as upper, middle and lower accordingly. For visualization of the posterior acoustic shadowing of the stone, focal zones were always placed at the depth of or slightly deeper than the stone level, with careful control of the B-mode gain setting. Color Doppler was applied over the site of the stone and twinkling sign was observed, a red and blue color map was used. The color window size was adjusted to cover the concerned area and adjacent tissue. The color Doppler gain was set to the point just below the threshold for color noise. When the twinkling sign was clearly obtained, power Doppler was also used to differentiate between the surrounding vessels and the ureter. In addition, color Doppler also helped in differentiation between stasis and prominent vessels at the renal hilum in certain cases. If there was no evidence of stones by Doppler ultrasound, then the patient was submitted to helical CT for further evaluation. The patients were followed up for a period of 7-8 weeks. A definite diagnosis was made when the patient passed a stone either spontaneously or after intervention.

\section{Statistical analysis}

Age and sex of the patients, stone site, size and side in addition to urine analysis results were 
retrieved from the medical charts. Data were analyzed by using SPSS version 15.0 (SPSS, Chicago Illinois, USA). The correlation between diagnostic tools (KUB, US), patients' characteristics (age and sex) and stones characteristics (site, size and side) was performed by using chi-square test. A value of $\mathrm{p} \leq 0.05$ was considered statistically significant.

\section{RESULTS}

Out of 939 patients, there were 825 males $(87.9 \%)$ and 114 females (12.1\%). Male to female ratio was $7: 1$. Age range (mean $\pm \mathrm{SD}$ ) was 9-80 years $(37.9 \pm 11)$. Our study found that renal ultrasound (gray-scale) when used alone detected calculi in 615 cases (65.4\%) and after utilizing CDU with twinkling sign, the diagnosis was made with confidence in 935 cases $(99.6 \%)$ which is statistically significant $(\mathrm{p}<0.05)$ (Table-1) and the twinkling was consistent in all confirmed cases (Figures 1A, $1 \mathrm{~B}$ and 2). The overall sensitivity and specificity of CDU were 99.6\% and 100\% respectively. There were $4(0.4 \%)$ patients in whom the stones could not be found neither by gray scale or color Doppler ultrasound and were not seen in KUB but the clinical scenario of pain and microhematuria warranted the employment of helical CT. In the latter group, the stones were confirmed by helical CT. The stones were located in the lower ureter in all $4 \mathrm{pa}-$ tients and the size of stones ranged from $3-5 \mathrm{~mm}$. The KUB showed radiopaque stones in 503 (53.6\%) cases and no stones were visualized in 436 patients (46.4\%) (Table-2). Microhematuria was present in 835 cases (88.9\%) with specificity of $89.1 \%$, while in
Figure $1 \mathrm{~A}$ - Grey scale ultrasound of stone in the left middle ureter with posterior shadowing.

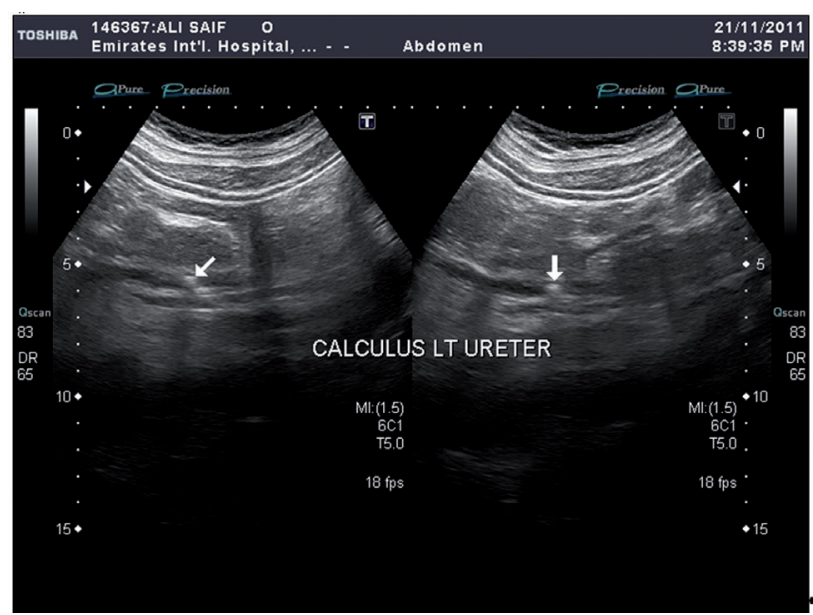

Figure 1B - Middle ureteral stone after application of color Doppler ultrasound with twinkling.

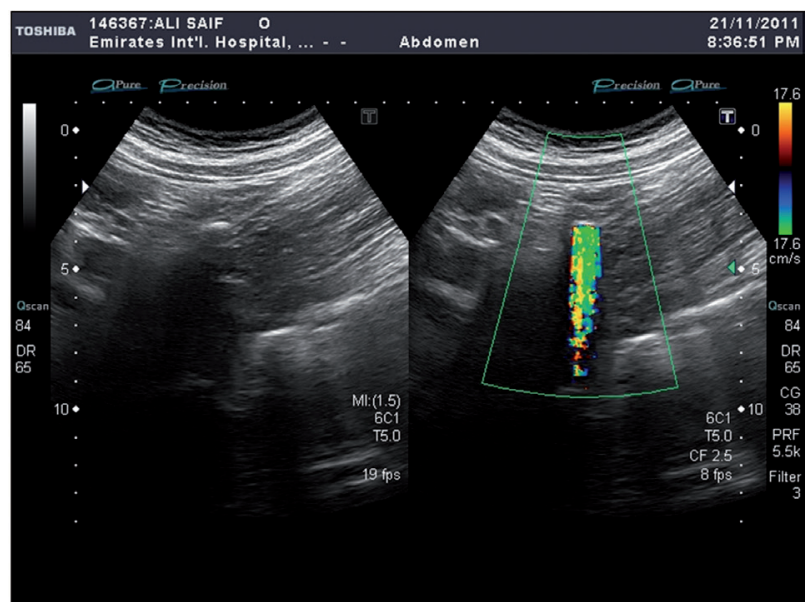

Table 1 - Detection rate and distribution of ureteral stones in 935 cases (bilateral in 4 cases) after comparison between gray scale ultrasound and color Doppler ultrasound.

\begin{tabular}{lcccc}
\hline & \multicolumn{2}{c}{ Gray-scale ultrasound } & \multicolumn{2}{c}{ Color Doppler ultrasound } \\
\hline Site & Number & $\%$ & Number & $\%$ \\
\hline Upper & 105 & $11.1 \%$ & 190 & $20.3 \%$ \\
Middle ureter & 30 & $43.1 \%$ & 77 & $8.2 \%$ \\
Lower & 480 & $51.2 \%$ & 672 & $71.5 \%$ \\
\hline Total & \multicolumn{2}{c}{$615(65.4 \%)$} & & $939(99.6 \%)$ \\
\hline
\end{tabular}


Figure 2 - Stone in the lower end of the ureter by gray scale ultrasound and the twinkling sign after the application of the color Doppler ultrasound.

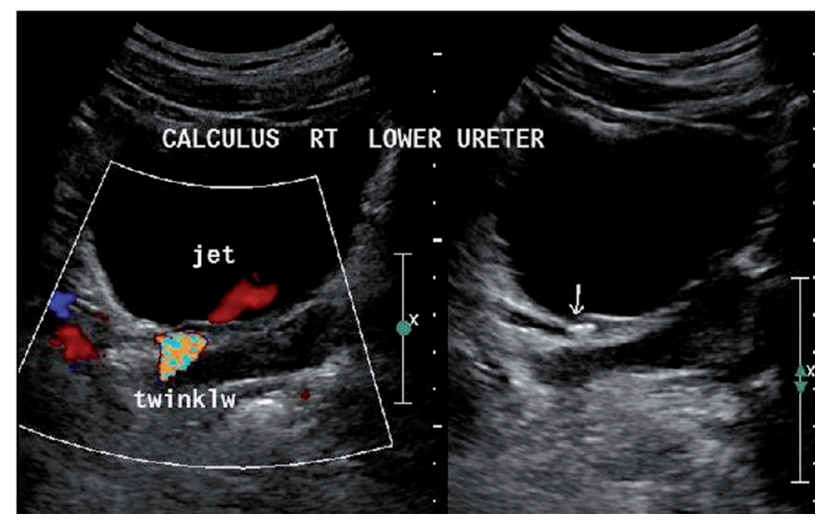

\section{DISCUSSION}

The diagnostic approach of acute flank pain is controversial and can vary from center to center, city to city or country to country depending on what is considered acceptable. Important factors include the local prevalence of stone disease, medical resources available, relative costs in a particular system, and the merits and limitations of each diagnostic modality (6). Although CT is the gold standard for diagnosis of renal colic, it is not available outside hospital facilities and is costly (7). There are recent concerns regarding the radiation exposure during CT examinations and its indiscriminate use. Moreover, many patients

Table 2 - Site and size of ureteral stones by KUB in 939 cases.

\begin{tabular}{lccccc}
\hline Site & Radiopaque & $\%$ & Radiolucent & $\%$ & $\begin{array}{c}\text { Mean stone } \\
\text { Size (mm) }\end{array}$ \\
\hline Upper & 139 & $77.6 \%$ & 51 & $11.7 \%$ & $11.7 \pm 1.8$ \\
Middle ureter & 42 & $8.3 \%$ & 35 & $8.1 \%$ & $7.1 \pm 0.8$ \\
Lower & 322 & $64.1 \%$ & 350 & $80.2 \%$ & $7.6 \pm 0.29$ \\
\hline \multicolumn{5}{c}{$503(53.6 \%)$} \\
\hline
\end{tabular}

102 cases (10.9\%) the urine was negative (Table-3) . There were 190 (20.3\%), 77 (8.2\%) and 671 (71.5\%) upper, middle and lower ureteral stones respectively (Table-1). Right side stones were seen in 438 $(46.7 \%)$ patients while left side stones were seen in 499 (53.3\%). Stones were detected bilaterally in 4 cases $(0.4 \%)$. The range of stones size (mean \pm $\mathrm{SD})$ was $3-26 \mathrm{~mm}(7.5 \pm 2.7)$. Simultaneous positive findings in US and KUB combined with microhematuria were found only in 453 (48.2\%) cases. US (gray and color Doppler) results were independent from any other factor while KUB results of stones were significantly related to site, side, size of stones, age of patient and urine analysis $(\mathrm{p}=0.000$, $0.042,0.005,0.000,0.016$ respectively). Microhematuria was related to stone size $(\mathrm{p}=0.008)$. may receive an additional radiation dose during follow-up studies (if a calculus is not expelled) or with new episode of colic and the cumulative effective doses of radiation from imaging procedures increased with advancing age $(8,9)$.

Although ultrasound is a safe diagnostic tool, medical literature data on its use in diagnosis of acute renal colic is quite heterogeneous with big difference between studies. Many authors reported that sonography has limited role in diagnosis of ureteral calculi, but these data were from the 1980s and 1990s and this opinion is conflicted by recent studies (10-12). There is a recent study that has reported the sensitivity and specificity of sonography, 93\% and 95\% respectively, by definite demonstration of lithiasis with new sonographic 
Table 3 - Relation between microhematuria and stone site in Color Doppler examined cases.

\begin{tabular}{|c|c|c|c|c|}
\hline Upper & 180 & $21.5 \%$ & 10 & $8.1 \%$ \\
\hline Middle ureter & 72 & $8.0 \%$ & 6 & $5.8 \%$ \\
\hline Lower & 583 & $69.9 \%$ & 86 & $84.1 \%$ \\
\hline & \multicolumn{2}{|c|}{ Positive 835 (88.9\%) } & \multicolumn{2}{|c|}{ Negative 102 (10.9\%) } \\
\hline
\end{tabular}

equipments and technologies (13).In our study, gray-scale ultrasound examination alone detected ureteral calculi in $65.4 \%$ of patients presented to ER with low specificity. The twinkling artifact was first described in 1996 by Rahmouni et al., as a sign generated by a highly reflective object that, despite being stationary, would generate a rapid alteration between red and blue when interrogated with color Doppler sonography (4). Although it was first described many years ago, the diagnostic value of twinkling in renal lithiasis is generally unacknowledged $(14,15)$. In the present study, the gray-scale Ultrasound sensitivity and specificity increased significantly when CDU was used with twinkling, where $99.6 \%$ of stones could be located with great confidence which is similar to results of recent studies $(5,9)$. Regarding the echographic identification of ureterolithiasis, there are numerous studies with quite contradictory results and with a very wide range of values, the sensitivity of the method ranging between 19\% and 96\% (1618). Our study showed the echographic identifications of ureteral calculi in $65.4 \%$ only. As there are different stone sizes and shapes, the twinkling sign can not differentiate between different types of stones albeit the results were consistent and independent of stones sizes. In 4 cases of our study (false negative), localization of ureteral stones could not be defined neither after using gray scale nor application of CDU, afterward helical CT localized the stones at lower ureter. These patients were obese and their BMI was over 35. Similarly, Lee et al. reported that 4 of 20 renal stones and 2 of 16 ureteral stones did not show any twinkling sign. The authors suggested that ureteral stones may be influenced more than renal stones by ultrasonic attenuation of interposed tissues because the ureter is deeply seated below abundant fat- ty tissue without a proper acoustic window (19). Others suggested reservation of CT for those patients who first have negative or equivocal results for KUB and sonography and this notion was applied in some of our cases (20). The size of stones in our study ranged from small sized to large ones (3-26mm) with twinkling artifact consistently generated in 99.6\% of cases which was independent of stone size and site. In the same way, Park et al. have documented the usefulness of the twinkling artifact in confirming the presence of small stones in $86 \%$ of cases (9). The application of CDU with twinkling needed a systematic screening of the ureter from the renal pelvis till the vesicoureteral junction with graded compression on the ureter especially if the stone is suspected in the mid-ureter or at the crossing of iliac vessels to reduce the distance between transducer and the ureter. This maneuver is similar to Puylaert technique of graduated compression in diagnosis of acute appendicitis (21). In recent studies, one group waited for 24 hours then scanned the patient, another study prepared the patient before ultrasound examination with intravenous drip infusion $(5,9)$. Conversely, in our series the patients were scanned within 2 hours of presentation to ER without special preparation or specific protocols of hydration. Therefore, this uniformity of ultrasound examination minimized the possibility of changes in stone location or the degree of ureteral dilatation that could affect the twinkling artifact quality and detection. Moreover, we did not find any association between the location of the stone and twinkling genesis. The twinkling generation was not related to ureteral dilatation or hydronephrosis. Lee et al. have reported the same conclusion (19).

In our study we found that $20.3 \%$ of the stones were located in upper ureter and only 8.2\% 
in the middle ureter while most (71.5\%) were situated in the lower ureter. Saita et al. determined the success rates of US according to the localization of the stone and they reported success rates of $82.2 \%$ in the proximal and 68\% in the distal ureter (20). Other authors reported that approximately 65\% of acutely presenting calculi impact in the lower segment of the ureter, therefore US has the potential to provide diagnostic follow up in a substantial number of individuals (22). Interestingly, we have used three ultrasound machines of different models and the results of twinkling artifact were reproducible. Although Aytac et al. (15) reported that the twinkling sign depends on the color sensitivity and the acoustic output of the ultrasound unit, we did not notice such observation.

KUB, when used alone in renal colic, is of limited diagnostic value with a sensitivity of 53$62 \%$ and specificity of $67-69 \%$ for the detection of ureteral calculi; in our series KUB detected radiopaque stones in 53.6\% of renal colic cases which is similar to others $(23,24)$. KUB was sufficient to document the size and site of radiopaque calculi as upper ureteral stones were more common than lower ureteral stones in this study $(\mathrm{p}<0.001)$. However, $46.4 \%$ of stones were not visible in $\mathrm{X}$-ray of our cases. KUB in young patients was significantly better in detection of stones than older age group $(p=0.005)$. This improvement may be either due to less soft tissue density or stone types but its significance should be weighed against expenses of radiation exposure.

Urinalysis has been widely accepted as a standard test for diagnosing acute renal colic, unlikely the incidence of negative hematuria in patients with ureterolithiasis has been reported to be $9 \%$ to $33 \%(25,26)$. In our study, microhematuria was detected in $88.9 \%$ of patients and absent in 10.9\%. We also have observed that microscopic hematuria was common with lower ureteral stones (69.6\%) and was related to stone size and noteworthy, was common with radiopaque than radiolucent stones $(\mathrm{p}=$ 0.016). Interestingly, in this retrospective study the urinalysis was requested at the initial presentation of renal colic to minimize possibility of false negative results since other authors found the incidence of negative hematuria is highest on the days 3 and 4 after initial presentation of renal colic (27).
Finally, there are some limitations of our study; it is a retrospective with its inherited shortcomings, the ultrasound did not show the association between the twinkling signs and the type of the stones, lastly we did not report on the outcome of renal colic cases despite the sufficient period of follow-up. However, our study was designed to determine the value of CDU with twinkling in the initial diagnosis of acute renal colic and is the largest cohort of renal colic patients diagnosed by using this technique. We feel that these encouraging results are of potential usefulness in the clinical practice with less cost, easy availability and mobility of ultrasound.

\section{CONCLUSIONS}

The present study emphasized that utilization of color Doppler ultrasound with twinkling in trained hands can provide an excellent alternative modality with high sensitivity and specificity in diagnosis of acute renal colic and should be employed at initial presentation in ER.

\section{ABBREVIATIONS}

KUB = Kidney, Ureter, Bladder

$\mathrm{CDU}=$ Color Doppler Ultrasound

$\mathrm{CT}=$ Computed Tomography

$\mathrm{ER}=$ Emergency Room

\section{CONFLICT OF INTEREST}

None declared.

\section{REFERENCES}

1. Ahmad NA, Ather MH, Rees J: Unenhanced helical computed tomography in the evaluation of acute flank pain. Int J Urol. 2003; 10: 287-92.

2. Smith RC, Levine J, Dalrymple NC, Barish M, Rosenfield AT: Acute flank pain: a modern approach to diagnosis and management. Semin Ultrasound CT MR. 1999; 20: 108-35.

3. Ferrandino MN, Bagrodia A, Pierre SA, Scales CD Jr, Rampersaud E, Pearle MS, et al.: Radiation exposure in the acute and short-term management of urolithiasis at 2 academic centers. J Urol. 2009; 181: 668-72; discussion 673. 
4. Rahmouni A, Bargoin R, Herment A, Bargoin N, Vasile N Color Doppler twinkling artifact in hyperechoic regions. Radiology. 1996; 199: 269-71.

5. Mitterberger M, Aigner F, Pallwein L, Pinggera GM, Neururer $\mathrm{R}$, Rehder $\mathrm{P}$, et al.: Sonographic detection of renal and ureteral stones. Value of the twinkling sign. Int Braz J Urol. 2009; 35: 532-9; discussion 540-1.

6. Shokeir AA, Abdulmaaboud M: Prospective comparison of nonenhanced helical computerized tomography and Doppler ultrasonography for the diagnosis of renal colic. J Urol. 2001; 165: 1082-4.

7. Grisi G, Stacul F, Cuttin R, Rimondini A, Meduri S, Dalla Palma L: Cost analysis of different protocols for imaging a patient with acute flank pain. Eur Radiol. 2000; 10: 1620-7.

8. Levine JA, Neitlich J, Verga M, Dalrymple N, Smith RC; Radiology: Ureteral calculi in patients with flank pain: correlation of plain radiography with unenhanced helical CT. 1997; 204: 27-31.

9. Park SJ, Yi BH, Lee HK, Kim YH, Kim GJ, Kim HC: Evaluation of patients with suspected ureteral calculi using sonography as an initial diagnostic tool: how can we improve diagnostic accuracy? J Ultrasound Med. 2008; 27: 1441-50.

10. Aslaksen A, Göthlin JH: Ultrasonic diagnosis of ureteral calculi in patients with acute flank pain. Eur J Radiol. 1990; 11: $87-90$.

11. Katz DS, Lane MJ, Sommer FG: Non-contrast spiral CT for patients with suspected renal colic. Eur Radiol. 1997; 7: 680-5.

12. Assi Z, Platt JF, Francis IR, Cohan RH, Korobkin M: Sensitivity of CT scout radiography and abdominal radiography for revealing ureteral calculi on helical CT: implications for radiologic follow-up. AJR Am J Roentgenol. 2000; 175: 333-7.

13. Patlas M, Farkas A, Fisher D, Zaghal I, Hadas-Halpern I: Ultrasound vs CT for the detection of ureteric stones in patients with renal colic. Br J Radiol. 2001; 74: 901-4.

14. Kamaya A, Tuthill T, Rubin JM: Twinkling artifact on color Doppler sonography: dependence on machine parameters and underlying cause. AJR Am J Roentgenol. 2003; 180: 215-22. Erratum in: AJR Am J Roentgenol. 2003; 180: 54.

15. Aytaç SK, Ozcan H: Effect of color Doppler system on the twinkling sign associated with urinary tract calculi. J Clin Ultrasound. 1999; 27: 433-9.

16. Yilmaz S, Sindel T, Arslan G, Ozkaynak C, Karaali K, Kabaalioğlu A, et al.: Renal colic: comparison of spiral CT, US and IVU in the detection of ureteral calculi. Eur Radiol. 1998; 8: 212-7.
17. Ripollés T, Agramunt M, Errando J, Martínez MJ, Coronel B, Morales M: Suspected ureteral colic: plain film and sonography vs unenhanced helical CT. A prospective study in 66 patients. Eur Radiol. 2004; 14: 129-36.

18. Sheafor DH, Hertzberg BS, Freed KS, Carroll BA, Keogan MT, Paulson EK, et al.: Nonenhanced helical CT and US in the emergency evaluation of patients with renal colic: prospective comparison. Radiology. 2000; 217: 792-7.

19. Lee JY, Kim SH, Cho JY, Han D: Color and power Doppler twinkling artifacts from urinary stones: clinical observations and phantom studies. AJR Am J Roentgenol. 2001; 176: 1441-5.

20. Saita $H$, Matsukawa M, Fukushima $H$, Ohyama $C$, Nagata $Y$ : Ultrasound diagnosis of ureteral stones: its usefulness with subsequent excretory urography. J Urol. 1988; 140: 28-31.

21. Puylaert JB: Acute appendicitis: US evaluation using graded compression. Radiology. 1986; 158: 355-60.

22. Eisner BH, Reese A, Sheth S, Stoller ML: Ureteral stone location at emergency room presentation with colic. J Urol. 2009; 182: 165-8.

23. Dalla Palma L, Stacul F, Bazzocchi M, Pagnan L, Festini G, Marega D: Ultrasonography and plain film versus intravenous urography in ureteric colic. Clin Radiol. 1993; 47: 333-6.

24. Svedström E, Alanen A, Nurmi M: Radiologic diagnosis of renal colic: the role of plain films, excretory urography and sonography. Eur J Radiol. 1990; 11: 180-3.

25. Press SM, Smith AD: Incidence of negative hematuria in patients with acute urinary lithiasis presenting to the emergency room with flank pain. Urology. 1995; 45: 753-7.

26. Li J, Kennedy D, Levine M, Kumar A, Mullen J: Absent hematuria and expensive computerized tomography: case characteristics of emergency urolithiasis. J Urol. 2001; 165: 782-4.

27. Kobayashi T, Nishizawa K, Mitsumori K, Ogura K: Impact of date of onset on the absence of hematuria in patients with acute renal colic. J Urol. 2003; 170(4 Pt 1): 1093-6.

Correspondence address: Mahmoud Abdel-Gawad, MD Consultant Urologist

Head of Surgical and Anesthesia Departments Emirates International Hospital Al Ain, P. Box: 18088, United Arab Emirates Fax: + 9713 763-6555

E-mail: naggarm@yahoo.com 